\title{
Experimental test of spontaneous collapse models with mechanical systems
}

\author{
Andrea Vinante ${ }^{* \dagger}$ \\ FBK-CNR Istituto di Fotonica e Nanotecnologie, Trento (Italy) and University of Trento (Italy) \\ E-mail: andrea.mistervin@gmail.com
}

\begin{abstract}
Spontaneous collapse models have been proposed as a possible realist solution of the quantum measurement problem. They assume that the standard unitary evolution is valid only in the microscopic limit, with stochastic corrections leading to a dynamical reduction of the wave function at macroscopic level. A remarkable feature of collapse models is that, unlike interpretations of quantum mechanics, they predict departures from standard theory that can be experimentally tested. A common prediction is a violation of energy conservation, which manifests as an universal force noise acting on mechanical systems. Here, we will discuss recent bounds on collapse models set by mechanical experiments, focusing on ultracold cantilevers and gravitational wave detectors. First notable results are a partial exclusion of the CSL parameters proposed by Adler and a full exclusion of an early model based on quantum gravity arguments.
\end{abstract}

Corfu Summer Institute 2017 "School and Workshops on Elementary Particle Physics and Gravity" 2-28 September 2017

Corfu, Greece

\footnotetext{
* Speaker.

${ }^{\dagger}$ Current affiliation: University of Southampton, UK
} 


\section{Introduction}

Spontaneous wave function collapse (or dynamical reduction) models [1, 2, 3, 4] have been proposed to reconcile the linear and deterministic evolution of quantum mechanics with the nonlinear and stochastic character of the measurement process. According to such models, random collapses occur spontaneously in any material system regardless of measurements occurring or not, leading to a progressive spatial localization of the wave function. The collapse rate scales with the size (number of constituents) of the system, in such a way as to produce rapid localization of any macroscopic system, while giving no measurable effect at the microscopic level, where standard quantum mechanics holds. Importantly, collapse models lead to a natural solution of the measurement problem, by predicting the emergence of well-defined outcomes in any measurement process in agreement with the Born rule.

The most studied and general collapse model is the so called Continuous Spontaneous Localization (CSL) [2]. It can be regarded as a continuous version of the earlier collapse model introduced in 1986 by Ghirardi, Rimini and Weber (GRW) [1], and solves some technical issues of the latter. CSL is characterized by two phenomenological constants, a collapse rate $\lambda$ and a characteristic length $r_{C}$, which characterize respectively the intensity and the spatial resolution of the spontaneous collapse process. $\lambda$ and $r_{C}$ are free parameters which should be derived, or bounded, by experiments. Other relevant collapse models are the Diosi-Penrose gravitational model $[5,6,7,8]$ and quantum gravity inspired models $[9,10]$.

The direct effect of collapse models such as CSL is to destroy quantum superpositions, resulting in a loss of coherence in interferometric matter-wave experiments [11, 12, 13]. However, indirect effects survive even at semiclassical level. The noise field associated with the collapse leads unavoidably to the violation of the energy conservation. So called non-interferometric tests have been thus proposed to look for these effects. Such tests include spontaneous emission of x-rays $[14,15]$, spontaneous heating and force noise in mechanical systems [16, 17, 18, 19, 20, 21, 22, 23] and spontaneous heating of ultracold atoms [24, 25].

In this paper, I will review recent experimental progress in testing collapse models by means of non-interferometric mechanical tests and discuss possible future developments.

\section{Models}

\subsection{The CSL model}

The CSL master equation can be written as [3]:

$$
\frac{\mathrm{d}}{\mathrm{d} t} \hat{\rho}(t)=-\frac{i}{\hbar}[\hat{H}, \hat{\rho}(t)]+\mathscr{L}_{\mathrm{CSL}}[\hat{\rho}(t)]
$$

where $\hat{H}$ describes the free evolution of the system. The CSL effect is described by a Lindblad type term $\mathscr{L}[\hat{\rho}(t)]$. In the case of a rigid body undergoing small displacements in a given direction $z$, with the motion described in terms of the center of mass coordinate, the CSL term can be written as [20]:

$$
\mathscr{L}_{\mathrm{CSL}}\left[\hat{\rho}_{\mathrm{CM}}(t)\right]=-\eta\left[\hat{q},\left[\hat{q}, \hat{\rho}_{\mathrm{CM}}(t)\right]\right]
$$


$\hat{q}=\hat{z}$ being the position operator of the center-of-mass, and:

$$
\begin{aligned}
\eta & =\frac{2 \lambda}{m_{0}^{2}} \iint \mathrm{d}^{3} \mathbf{r} \mathrm{d}^{3} \mathbf{r}^{\prime} \exp \left(-\frac{\left|\mathbf{r}-\mathbf{r}^{\prime}\right|^{2}}{4 r_{C}^{2}}\right) \frac{\partial \rho(\mathbf{r})}{\partial z} \frac{\partial \rho\left(\mathbf{r}^{\prime}\right)}{\partial z^{\prime}} \\
& =\frac{(4 \pi)^{\frac{3}{2}} \lambda r_{C}^{3}}{m_{0}^{2}} \int \frac{\mathrm{d}^{3} \mathbf{k}}{(2 \pi)^{3}} k_{z}^{2} e^{-\mathbf{k}^{2} r_{C}^{2}}|\tilde{\rho}(\mathbf{k})|^{2}
\end{aligned}
$$

with $\mathbf{k}=\left(k_{x}, k_{y}, k_{z}\right), \tilde{\rho}(\mathbf{k})=\int \mathrm{d}^{3} \mathbf{x} e^{i \mathbf{k} \cdot \mathbf{r}} \rho(\mathbf{r})$ and $\rho(\mathbf{r})$ the mass density distribution of the system. In the expressions above $m_{0}$ is the nucleon mass, $\lambda$ and $r_{C}$ are free parameters. The CSL term causes a collapse of the wave function in the position coordinate, i.e. a localization of the center of mass, and therefore a loss of quantum coherence as an effect of the collapse process.

The CSL dynamics is characterized by two phenomenological parameters: the collapse rate $\lambda$, which represents the rate of localization of a single nucleon, and the characteristic length $r_{C}$, which is related to a smearing of the mass-density function. These are free parameters of the model which should be derived, or bounded, by experiments. The standard conservative values suggested by GRW are $\lambda \simeq 10^{-16} \mathrm{~s}^{-1}$ and $r_{C}=10^{-7} \mathrm{~m}[1,2]$ and are sufficient to guarantee almost instantaneous localization of macroscopic objects. A strongly enhanced value for the collapse rate has been suggested by Adler [26], motivated by the requirement of making the wave function collapse effective at the level of latent image formation in photographic process. The values suggested by Adler are $\sim 10^{9 \pm 2}$ times larger than standard values at $r_{C}=10^{-7} \mathrm{~m}$, and $\sim 10^{11 \pm 2}$ times larger at $r_{C}=10^{-6} \mathrm{~m}$.

Besides causing localization, the CSL term expressed by Eq. (2.2) describes a diffusion of the center of mass, and $\eta$ can be interpreted as diffusion constant $[16,17]$. In other words, the center of mass of rigid body will undergo a brownian motion due to the action of the continuous collapselocalization process. This consideration is at the base of so called non-interferometric methods to look for CSL. More generally, the existence of a fundamental CSL collapse mechanism necessarily implies the appearance of a universal force noise, acting on any massive object, with (one-sided) power spectral density $S_{f f}=2 \hbar^{2} \eta$. A simple consequence is that for a mechanical resonator in equilibrium with a thermal bath at temperature $T$, the mean energy is given by [20,21]:

$$
\langle E\rangle=k_{B} T+\frac{\hbar^{2} \eta Q}{2 m \omega_{0}}
$$

i.e. CSL predicts a net excess of energy with respect to the classical equipartition value.

Experimentally, there are two similar and almost equivalent approaches to look for CSLinduced effects. In the simplest one, a mechanical resonator is continuously monitored and its mean energy is measured as a function of temperature. The equipartition term $k_{B} T$ can be characterized and subtracted, and the hypothetical CSL term can be estimated or bounded. The second approach is slightly more general as it applies to any system, even to a free mass. By characterizing and monitoring the free dynamics of the system one infers the power spectral density of the force noise acting on it, which is given, in the thermal limit $k_{B} T \gg \hbar \omega$ and in absence of technical noise, by:

$$
S_{f f}=\frac{4 k_{B} T m \omega}{Q}+2 \hbar^{2} \eta .
$$


Accurate measurements as a function of the temperature $T$ and the damping factor $\gamma=m \omega / Q$, which must be independently measured, allow to characterize and subtract the thermal term and to estimate or bound the collapse-noise term.

In both situations, in order to increase the sensitivity to CSL noise, one has to maximize the 'noise to noise' ratio between the CSL term and the thermal noise. In practice this means lowest possible temperature $T$, highest possible time constant $\tau=Q / \omega$, and highest possible $\eta / m$ ratio. While the first two conditions merely express the requirement of lowest possible rate of energy exchange with the thermal bath, the third condition leads to consequences which are peculiar to the model details. For CSL we can distinguish two relevant limits. When the characteristic size $L$ of the system is small, $L \ll r_{C}$, then one can show that $\eta \propto m^{2}$ and thus $\eta / m \propto m$. When the characteristic length of the system in the direction of motion $L$ is large, $L \gg r_{C}$, then $\eta \propto \rho^{2} A$ and thus $\eta / m \propto \rho / L$, where $\rho$ is the mass density and $A$ is the area transverse to the motion [20,21]. The expressions in the two limits imply that, for a well defined characteristic length $r_{C}$, the optimal system is a plate or disk with thickness $L \sim r_{C}$ and the largest possible density $\rho$.

\subsection{The Diosi-Penrose model}

While CSL is the most general and perhaps the most studied collapse model, other models have been proposed, attempting to provide a connection of the wave function collapse to specific mechanisms, in particular to gravity. There are several motivations behind this idea. On the one hand, gravity is the only known force that we have not been able to merge with quantum theory, despite enormous theoretical efforts. Indeed, there is not even an absolutely compelling evidence that gravity must be quantized. On the other hand, collapse models suggest that a possible breakdown of the quantum superposition principle has to be linked to a mass-proportional localizing mechanism. This points to gravity as a natural candidate.

Among the supporters of this point of view, Penrose argues that the quantum superposition principle is in fundamental contradiction with the general covariance principle of general relativity $[5,6]$. In open contrast with the standard point of view that gravity has to be quantized, he argues that superpositions of a massive object in different spatial position can survive only for a finite time $\tau \sim \hbar / \Delta E$ where $\Delta E$ is a properly defined difference between the self-gravitational energies associated to the two states. In other words, the quantum superposition principle must be broken at fundamental level by gravity.

However, Penrose argument is heuristic and does not provide a dynamical description of gravitationally induced collapse. An attempt in this direction has been done by Diosi [7]. His model is rather similar to CSL, with a different kernel for the the localization integral. The intensity of the collapse rate would be determined by gravity, while a phenomenological length scale $r_{D P}$, analogous to $r_{C}$, is necessary to avoid exceedingly large violations of energy conservation [8]. Experimentally, values of $r_{D P}$ as low as the nuclear size $10^{-14} \mathrm{~m}$ are in priciple allowed, although values $r_{D P} \sim r_{C}=10^{-7} \mathrm{~m}$ have been suggested as more reasonable [8]. Notably, the collapse time scale predicted by Diosi coincides with the one heuristically suggested by Penrose, which is the reason motivating the denomination of Diosi-Penrose (DP) model in literature.

The DP model leads to localization and diffusion similar to CSL. The diffusion constant $\eta_{D P}$ 
is given by [21]:

$$
\eta_{D P}=\frac{G \rho m}{6 \sqrt{\pi} \hbar}\left(\frac{a}{r_{D P}}\right)^{3},
$$

where $a$ is the lattice constant and $G$ is the gravitational constant.

Experimentally, Eqs. (2.5) and (2.6) hold exactly in the DP model as in CSL, by replacing $\eta$ with $\eta_{D P}$. The requirements of lowest possible $T$ and longest possible time constant $\tau=Q / \omega$ remain unchanged. A notable difference from CSL is that the diffusion constant $\eta_{D P}$ does not show any shape dependence. In particular, for the relevant case of large size $L \gg r_{D P}$, the ratio $\eta_{D P} / m$, which determines the experimental sensitivity, depends only on the density. In other words there is no optimal size or shape.

Another peculiarity of the DP model is that it is characterized by a single free parameter. While a given null experiment will exclude a given region of the CSL two-parameter space $\lambda-r_{C}$, in the DP case it will set a lower bound on the $r_{D P}$ parameter.

\subsection{The Ellis model}

We briefly mention another collapse model proposed in literature which is suitable for current experimental tests. The model, proposed by Ellis and coworkers [9, 10], is based on quantum gravity considerations. The wave function of a massive object would be effectively localized by the interaction with a bath of quantum wormholes which characterize the spacetime structure at the Planck length scale. As such, the Ellis model is not strictly speaking a collapse model, rather it is a decoherence model, where decoherence is induced by practically unaccessible degrees of freedom.

As long as the wavelength of wormholes is much longer than the characteristic magnitude of the motion of the system, decoherence can be effectively described by Eq. (2.2), with diffusion constant [27]:

$$
\eta_{E}=\frac{\left(m_{0} c\right)^{4} m^{2}}{\left(\hbar m_{P l}\right)^{3}}
$$

where $m_{P l}$ is the Planck mass and $c$ is the speed of light. Compared to CSL and DP models, the Ellis model has the merit of being parameter-free, which allows for unambiguous experimental falsifiability.

\section{Experiments}

\subsection{Overview}

There are at least three areas of current experimental research that deal with ultrasensitive measurements with mechanical resonators: (a) force microscopy technologies; (b) macroscopic experiments to detect weak forces, such as gravitational wave detectors and torsion pendulums; (c) quantum optomechanical systems.

Force microscopy technologies are nowadays a standard tool in applied physics and surface science. The invention of atomic force microscopy (AFM) [28] has been awarded by the Nobel Prize. AFM is based on very sensitive cantilevers, featuring atomically sharp tip, which can be used to map the surface of a sample with atomic resolution by detecting the tip-surface force. One of the most extreme versions of force microscopy is the so called magnetic resonance force 
microscopy (MRFM), which combines magnetic resonance imaging with ultrasensitive microcantilevers to yield three-dimensional maps of a sample with nanometer resolution. This technique is very demanding, and has led to the development of very sensitive nanocantilevers with relatively low frequency in the $\mathrm{kHz}$ range, high quality factor, and to their operation at very low temperature down to the millikelvin regime [29]. According to Eqs. (2.5) and (2.6) these systems are natural candidates to detect collapse-induced noise. Furthemore, their size compares favourably with the theoretically suggested CSL length scale $[2,26]$.

Macroscopic experiments such as torsion pendulums and gravitational wave detectors [30, 31] are by design force sensors with exquisitely low force noise. The first observation of gravitational waves by LIGO has been awarded by the Nobel Prize. These experiments consist of macroscopic mechanical systems with high quality factor and typically very low frequency, in the $\mathrm{Hz}$ or sub- $\mathrm{Hz}$ range. According to Eqs. (2.5) and (2.6) they are obvious candidates to detect or bound collapseinduced force noise. The main strong feature with respect to other systems is the operation at low frequency, which requires extreme care in mechanical isolation from environmental noise. The only limitations come from being macroscopic and thus far from the theoretically suggested CSL length scale (CSL, adler), and from the fact that these experiments are normally operated at room temperature. However, resonant bar gravitational wave detectors [31] and prototype of future interferometric detectors have been operated at cryogenic temperature as well.

Quantum optomechanical systems (QOS) are also in principle good candidates to detect weak forces [32]. Although the key ideas are historically derived from gravitational wave detectors (and the latters are in fact very special QOS) modern QOS are optimized for a different goal, which is bringing a mechanical resonator in the quantum regime and exploiting its properties the context of quantum technologies. The key ingredients to achieve the quantum regime are low $T$, high $Q$ and high frequency $\omega$. Thus, while the requirement to minimize the $T / Q$ factor is the same as in weak force detection, the natural tendency in quantum optomechanics is to push for very high frequency up to the GHz regime, which increases the zero-point energy. This runs against the requirement of low frequency expressed by Eqs. (2.5) and (2.6). In fact, despite the explosion of the field in the last decade, the incredible variety of quantum optomechanical systems, and the impressive results obtained so far [32], the bounds on collapse models that can be inferred from QOS are usually not as strong as with the other approaches.

In the next section we will mainly discuss the bounds on collapse models that can be inferred from ultracold cantilevers based on force microscopy technologies and from macroscopic experiments.

\subsection{Ultracold cantilevers}

The first serious attempt to exploit mechanical resonators to bound the parameters of collapse models, in particular the CSL model, was done by Vinante et al. in 2016 [33]. The system described in the latter work is a MRFM nanocantilever cooled to temperatures down to $10 \mathrm{mK}$. A magnetic microsphere was attached to the cantilever free end to allow low noise magnetic detection using a SQUID. The original motivation of this solution was to provide a way to operate a MRFM nanocantilever at millikelvin temperature avoiding the overheating typical of laser-based detection. This goal was successfully achieved, as the cantilever thermal noise was found in good agreement with the equipartition theorem down to $25 \mathrm{mK}$ [29]. From this observation an upper bound on any 
non-thermal contribution, including hypothetical CSL noise, was estimated. An exclusion curve on the CSL parameter space was then derived (Fig. 1, orange line) [33].

The main notable result was the first partial exclusion of the CSL parameters suggested by Adler, in particular the full exclusion of the parameters suggested at $r_{C}=10^{-6}$. However the experiment was far from being optimized. The spherical geometry is not the one that maximizes the effect of CSL-induced noise, and the density of the material is three times lower than the densest known materials, such as osmium or iridium. But above all, the quality factor is this experiment, $Q \sim 10^{5}$ was not impressive.

A second similar experiment was reported more recently [34] with cantilever and magnetic sphere roughly 10 times larger than the previous one. The cantilever was a standard AFM cantilever, featuring a very large quality factor, up to $10^{7}$ at $20 \mathrm{mK}$. Very accurate measurements of the force noise were performed and, according to Eq. (2.6), the thermal contribution was characterized and subtracted. In contrast with the first experiment, a residual force noise was revealed. The ultimate origin of this noise is still unexplained. Further measurements have shown that it is not related to back-action from the SQUID and likely not due to vibrational noise.

For the purpose of testing CSL, the experiment is still able to enlarge the exclusion region. The exclusion line is shown in red in Fig. 1. If the source of excess noise were explained, the bound would be improved by one order of magnitude (dashed red line in Fig. 1). Of course, the excess noise could be in principle explained as CSL noise as well, and it would be compatible with the CSL effect predicted by Adler. However, given the latest data from LISA Pathfinder (see next section), this explanation seems very unlikely.

\subsection{Macroscopic experiments: LISA Pathfinder}

The bounds on collapse models that can be inferred by macroscopic experiments have been recently discussed by Carlesso et al. [27]. Different experiments have been considered: cryogenic resonant bar detectors of gravitational waves, interferometric detectors of gravitational waves (LIGO), torsion pendulums. It was finally shown that the strongest bounds on collapse models are set by the recently launched space mission LISA Pathfinder (LPF) [35], the technological demonstrator of the space-born gravitational wave detector LISA.

In LPF, two identical AuPt cubic test masses are nominally in free-fall in space, protected by a spacecraft which follows the masses in such way to minimize the residual coupling. The goal of the mission was to assess the achievable accuracy of free-fall, by optically measuring the residual relative acceleration noise (or equivalently force noise) between the two test masses. Thanks to the quiet space environment, it was possible to achieve an impressive low acceleration noise of 5.2 $\mathrm{fm} /\left(\mathrm{m} / \mathrm{s}^{2}\right) / \sqrt{\mathrm{Hz}}$ [35], recently improved by a factor of three following a progressive reduction of residual gas pressure with time [36].

LPF and the future LISA mission are optimized for extremely low frequencies in the range from $10^{-4} \mathrm{~Hz}$ to $10^{-1} \mathrm{~Hz}$. In this frequency range, LPF outperforms ground-based torsion pendulums by several orders of magnitude in force power spectral density. Ground based gravitational wave detectors such as Advanced LIGO, despite the impressive mechanical attenuation, are outperformed too by LPF in terms of force noise, mainly because of the higher characteristic frequency. In fact the minimum of the force noise in LIGO is around $30 \mathrm{~Hz}$ [27]. 


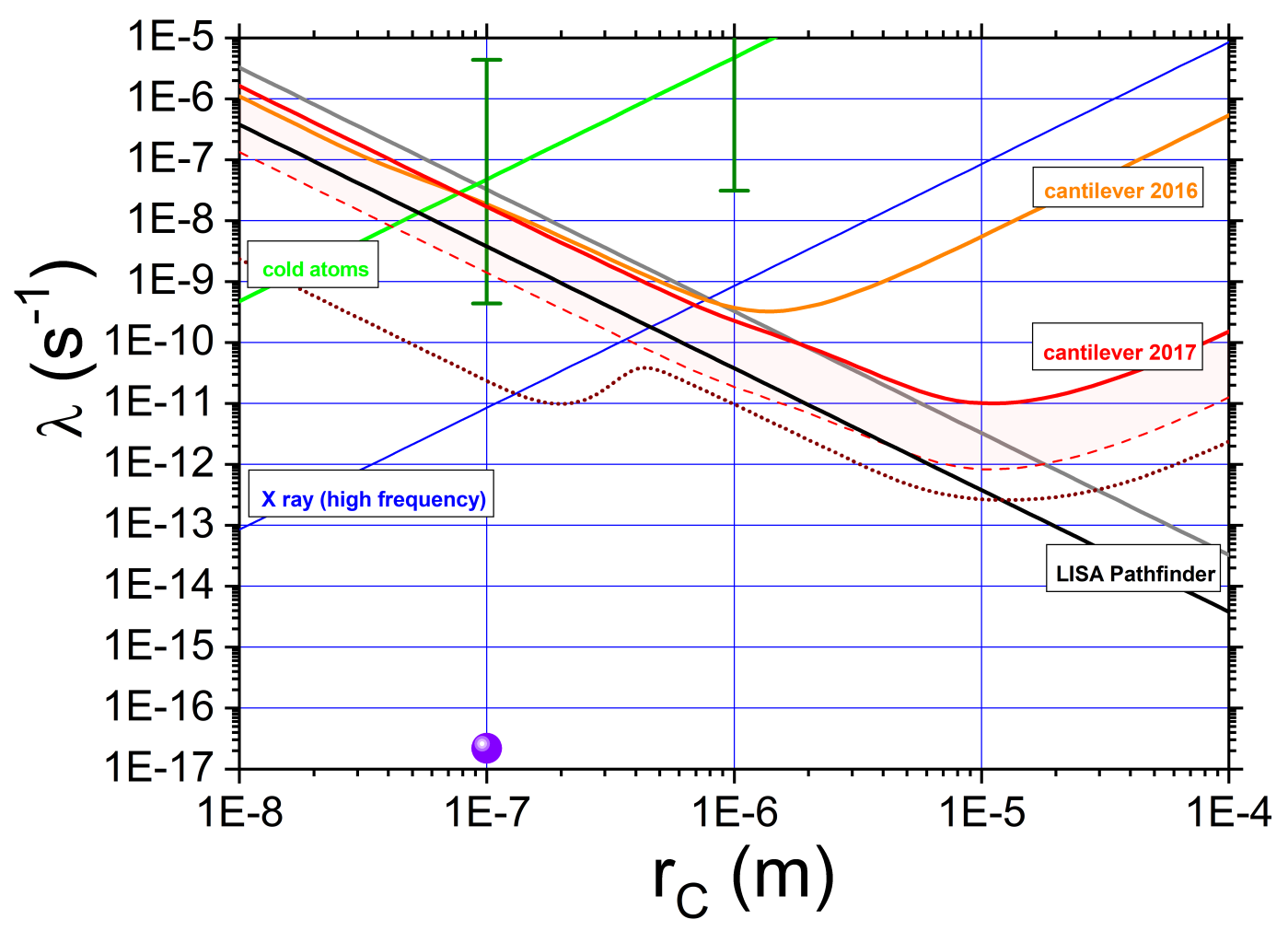

Figure 1: Upper limits on the CSL model collapse rate from different non-interferometric experiments. For each curve the region above the curve is excluded. X-ray spontaneous emission (blue) [15], cold atoms (green) [25], cantilever 2016 experiment (orange) [33], cantilever 2017 experiment (red) [34], LISA Pathfinder early data (gray) [27, 35] and final data (black) [36]. We also plot the potential improvement from the cantilever 2017 experiment if the excess noise were removed (dashed red line) and the projected upper limit from ongoing cantilever experiments with optimized test mass (wine dotted curve)). The theoretical parameters suggested by Adler (dark green bars) [26] and GRW (violet dot) [1] are also shown.

The bounds on the CSL model that can be set using LPF data are easily calculated using Eq. (2.6). As in LPF there is no absolute calibration of the thermal noise, one assumes the worst case condition and attributes the all measured noise to CSL. The gray and black curves in Fig. 1 refer respectively to the first data released officially [35] and the final data [36].

\subsection{Discussion}

The bounds on CSL obtained from ultracold cantilevers and LPF are reported in Fig. 1, together with other relevant bounds from other experimental techniques, such as spontaneous emission of x-ray [15] and spontaneous heating of ultracold atoms [25]. In addition, the CSL parameters suggested by Adler (dark green bars) [26] and GRW (violet dot) [1] are shown.

We remark that the bound from x-ray relies on the strong assumption that the CSL noise field extends up to $10^{18} \mathrm{~Hz}$. The bound may be easily evaded by a CSL noise field with a frequency cutoff. In contrast the bounds from mechanical systems and cold atoms probe the CSL noise field 
at low frequencies, and are therefore considered more significant. On the other hand, bounds from matter-wave interferometry, which represent direct test of the quantum superposition principle, are so far are at the level of $\lambda \sim 10^{-6} \mathrm{~s}^{-1}$ [37], thus far weaker than those inferred by mechanical resonators.

As shown in Fig. 1, current mechanical bounds are seriously challenging the predictions of the collapse rate by Adler, based on the assumption that the wave function collapse is effective already at the mesoscopic scale [26]. In contrast, the more conservative prediction by GRW is still many orders of magnitude off, and its testing will require impressive improvements on the experimental side.

It is an interesting fact that, at the end of the day, experiments with so huge difference in the relevant parameters, such as ultracold cantilevers and LPF, provide a comparable bound on CSL. We can explain this remarkable coincidence with the help of Eqs. (2.6). As discussed in the theory section, in the relevant limit $L \gg r_{C}$, the CSL over thermal noise ratio scales as $\rho Q /(T \omega L)$. Referring to the cantilever experiment, the LPF experiment provides roughly a $10^{1}$ times larger effective $Q$ and $10^{6}$ times lower $\omega$, giving 7 orders of magnitude improvement. However, the temperature $T$ and size $L$ are respectively $10^{4}$ and $10^{3}$ times larger, resulting in a nearly exact compensation by 7 orders of magnitude.

This gives also a clear indication of how the ultimate experiment to test CSL ought to be: very low frequency as in LPF, ultralow temperature and micrometer size as in the cantilever case. This calls for an experiment to be performed at ultralow temperature in a space environment.

Let us consider now the DP model. In this case, there is no optimal length scale, and consistently it is found that the strongest bound on the characterstic length $r_{D P}$ is set by LPF, roughly one order of magnitude better than cantilevers [38]. Specifically, using the final data of LPF [36], the lower bound $r_{D P}>83 \mathrm{fm}$ is found, implying that $r_{D P}$ must be larger than the size of any stable nuclei. This result does not appear so significant, for instance it is very far from the normalization length suggested by Ghirardi et al [8] of $10^{-7} \mathrm{~m}$. However, it rules out some earlier speculation that the nuclear size could possibly set the DP model length scale [21].

Finally, let us consider the Ellis model. It is possible to show that current LPF data, but data from other macroscopic experiments as well, are inconsistent with the Ellis model, as they would have already been able to point out the associated noise expressed by Eq. (2.8) [27]. Remarkably, the same conclusion has been recently obtained by analyzing atom interferometry experiments [39]. This is perhaps the first example of a collapse model which is completely ruled out by experiments.

\section{Outlook and conclusions}

We conclude with an outlook of possible near future and long term progress.

Concerning macroscopic experiments, a significant improvement within the next 15 years seems unlikely. The LISA Pathfinder mission is over, and the LISA mission is scheduled to be launched in the early 2030s. Moreover, as the LPF mission has already achieved the free-fall requirement for LISA it is not clear if LISA will be able to improve over LPF force noise significantly.

On the other hand, ground based experiment, such as advanced LIGO or similar detectors, are quite far from being significant with respect to bounding collapse models. Specifically, the upper limit on the CSL model is worse then the one from LPF by almost 4 orders of magnitude with the 
initial advanced LIGO data and 2 orders of magnitude with the final projected design sensitivity. However, the third generation of gravitational wave interferometers is expected to significantly improve the low frequency force noise, by means of cryogenic suspension and underground operation. This might perhaps allow to approach performance close to LPF.

In contrast, experiments with ultracold cantilevers or different micromechanical resonators have a better potential for improvement in the short-mid term, as they are far from being optimized. Improvements by 2-3 orders of magnitude appear possible in the near future by a combination of two main strategies. The first is moving to lower frequency, by means of softer resonators or larger mass. This will require a much better mechanical isolation, but entirely within reach. The second is an optimization of the test mass. In the first cantilever experiments the test mass was a magnetic sphere made of NdFeB. Far better results can be obtained by using disk geometries and high density materials such as Au or Pt as in LPF. Recent calculations show that the sensitivity to an hypothetical CSL noise field can be enhanced by using a multilayer test mass composed of alternate materials with high density contrast [40].

As an example we have plotted in Fig. 1 a dotted curve which shows the upper limit that could be obtained by the same cantilever used in Ref. [34] (red curve) with similar force noise, but with an optimized multilayered mass with $\mathrm{Au}$ or $\mathrm{W}$ as heavy material. This experiment, which would be incremental with respect to previous cantilever experiments, would completely rule out the CSL collapse rate suggested by Adler, and would outperform LPF over the whole relevant range of $r_{C}$ up to $10^{-5} \mathrm{~m}$. In addition, it would be able to completely rule out the excess noise observed in [34] as a possible CSL effect. Needless to say, any further reduction of the force noise, for instance by removing the excess noise or reducing the effective $T / Q$ factor, would further improve the bounds.

As the thermal noise is reduced, the quantum limit on the position measurement in these experiments will likely pose significant obstacles for improvements far beyond the proposed new cantilever experiment [20]. Possible ways to avoid the quantum limit are quantum non demolition strategies or stroboscopic measurements [22, 23].

A more radical approach towards a huge improvement is to replace the cantilever with a optical, electrical or magnetic trap, i.e. by levitating a nano or micromechanical test mass. Operation under extreme vacuum and ultralow temperature conditions might in principle allow effective quality factors exceeding $10^{12}$. However, we are far from a demonstration of this figure. Optomechanical levitation of nanoparticles is a hot topic of the latest years. For operation at very low temperature, electrical levitation by Paul traps or by magnetic levitation with superconductors appear much more suitable.

In order to exploit the extreme performance that may be achievable using levitated particles, space environment will be likely necessary, as in the LPF case. An ambitious program in this direction is the proposed space mission MAQRO [41].

\section{Acknowledgments}

The author acknowledges support from the International Centre for Theoretical Studies (ICTS) for the participation to the program Fundamental Problems in Quantum Physics (Code: ICTS/Progfpqp/2016/11), from EU through the COST Action MP1405, and from a Marie Curie Alumni Association (MCAA) Micro Travel Grant 2017. 


\section{References}

[1] G.C. Ghirardi, A. Rimini, and T. Weber, Phys. Rev. D 34, 470 (1986).

[2] G.C. Ghirardi, P. Pearle, and A. Rimini, Phys. Rev. A 42, 78 (1990); G. C. Ghirardi, R. Grassi, and F. Benatti, Found. Phys. 25, 5 (1995).

[3] A. Bassi, and G. C. Ghirardi, Phys. Rep. 379, 257 (2003).

[4] A. Bassi, K. Lochan, S. Satin, T. P. Singh, and H. Ulbricht, Rev. Mod. Phys. 85, 471 (2013).

[5] R. Penrose, Gen. Relativ. Gravit. 28, 581 (1996).

[6] R. Penrose, Phil. Trans. R. Soc. A 356, 1927 (1998).

[7] L. Diosi, Phys. Rev. A 40, 1165 (1989).

[8] G.C. Ghirardi, R. Grassi, and A. Rimini, Phys. Rev. A 42, 1057 (1990).

[9] J. Ellis, D.V. Nanopoulos and S. Mohanty, Nucl. Phys. B 241, 381 (1984).

[10] J. Ellis, S. Mohanty, and D. V. Nanopoulos, Phys. Lett. B 221, 113 (1989).

[11] K. Hornberger, S. Gerlich, P. Haslinger, S. Nimmrichter and M. Arndt, Rev. Mod. Phys. 84, 157 (2012).

[12] T. Juffmann, H. Ulbricht and M. Arndt, Rep. Prog. Phys. 76, 086402 (2013).

[13] M. Arndt and K. Hornberger, Nat. Phys. 10, 271 (2014).

[14] S.L. Adler and F. Ramanazoglu, Journ Phys. A 40, 13395 (2007).

[15] C. Curceanu, B.C. Hiesmayr, and K. Piscicchia, J. Adv. Phys. 4, 263 (2015).

[16] B. Collett and P. Pearle, Found. Phys. 33, 1495 (2003).

[17] S.L. Adler, J. Phys. A 38, 2729 (2005).

[18] A. Bassi, E. Ippoliti, S.L. Adler, Phys. Rev. Lett. 94, 030401 (2005).

[19] M. Bahrami, M. Paternostro, A. Bassi, and H. Ulbricht, Phys. Rev. Lett. 112, 210404 (2014).

[20] S. Nimmrichter, K. Hornberger, and K. Hammerer, Phys. Rev. Lett. 113, 020405 (2014).

[21] L. Diosi, Phys. Rev. Lett. 114, 050403 (2015).

[22] D. Goldwater, M. Paternostro, and P.F. Barker, Phys. Rev. A 94, 010104 (2016).

[23] J. Li, S. Zippilli, J. Zhang, and D. Vitali, Phys. Rev. A 93, 050102 (2016).

[24] T. Kovachy, J.M. Hogan, A. Sugarbaker, S.M. Dickerson, C.A. Donnelly, C. Overstreet, and M.A. Kasevich, Phys. Rev. Lett. 114, 143004 (2015).

[25] M. Bilardello, S. Donadi, A. Vinante, and A. Bassi, Physica A 462, 764 (2016).

[26] S.L. Adler, J. Phys. A 40, 2935 (2007).

[27] M. Carlesso, A. Bassi, P. Falferi, and A. Vinante, Phys. Rev. D 94, 124036 (2016).

[28] G. Binnig, C.F. Quate, and C. Gerber, Phys. Rev. Lett. bf 56, 930 (1986).

[29] O. Usenko, A. Vinante, G. Wijts, T.H. Oosterkamp, Appl. Phys. Lett. 98, 133105 (2011).

[30] B.P. Abbott et al (LIGO Scientific Collaboration and Virgo Collaboration), Phys. Rev. Lett. 116, 061102 (2016). 
[31] A. Vinante et al. (AURIGA Collaboration), Class. Quantum Grav. 23, S103 (2006).

[32] M. Aspelmeyer, T. Kippenberg, F. Marquardt, Rev. Mod. Phys. 86, 1391 (2014).

[33] A. Vinante, M. Bahrami, A. Bassi, O. Usenko, G. Wijts, T.H. Oosterkamp, Phys Rev. Lett. 116, 090402 (2016).

[34] A. Vinante, R. Mezzena, P. Falferi, M. Carlesso, A. Bassi, Phys. Rev. Lett. 119, 110401 (2017).

[35] M. Armano et al., Phys. Rev. Lett. 116, 231101 (2017).

[36] M. Armano et al., Phys. Rev. Lett. 120, 061101 (2018).

[37] M. Toros and A. Bassi, J. Phys. A: Math. Theor. 51, 115302 (2018).

[38] B. Helou, B. Slagmolen, D.E. McClelland, and Y. Chen, Phys. Rev. D 95, 084054 (2017).

[39] J. Minar, P. Sekatski, and N. Sangouard, Phys. Rev. A 94, 062111 (2016).

[40] A. Vinante et al., in preparation (2018).

[41] R. Kaltenbaek et al., Exp. Astron. 34, 123 (2012). 